\title{
Intrinsic exchange bias effect in strain-engineered single antiferromagnetic $\mathrm{LaMnO}_{3}$ films
}

\author{
Guowei Zhou ${ }^{1,2}$, Huihui Ji ${ }^{2}$, Yuhao Bai ${ }^{1,3}$, Zhiyong Quan ${ }^{1,2}$ and Xiaohong $\mathrm{Xu}^{1,2^{*}}$
}

\begin{abstract}
In this work, epitaxial growth of $\mathrm{LaMnO}_{3}$ thin films on different substrates using pulsed laser deposition under tensile and compressive strain was studied. The intrinsic exchange bias effect was observed in the single A-type antiferromagnetic $\mathrm{LaMnO}_{3}$ films no matter whether the tensile or compressive strain was supplied by the substrates. Due to the lattice mismatch between the film and different substrates, the intense strain can induce $\mathrm{MnO}_{6}$ octahedral rotation in the bottom region of the film neighboring the substrate, which leads to the distortion of $\mathrm{MnO}_{6}$ octahedron and the net magnetic behavior. However, the upper part maintains the original A-type antiferromagnetic order due to strain relaxation. The exchange bias effect in single films is attributed to the coupling between the bottom canted magnetic part and the upper antiferromagnetic region. The observation of exchange bias in single films on different substrates enables the emergence of a new class of biasing components in spintronics, which are based on strain-engineering.
\end{abstract}

Keywords: magnetic insulating state, exchange bias, $\mathrm{MnO}_{6}$ octahedral rotation, strain and interface effects, magnetic properties

\section{INTRODUCTION}

Perovskite structure oxides, which generate a rich spectrum of exotic properties, are known to constitute an interesting research topic in condensed-matter physics. The discovery of exchange bias (EB) effect by Meiklejohn and Bean has resulted in diverse tantalizing avenues as the electronic devices including various giant magnetoresistive sensors, magnetic recording read heads, and spin valves [1-3]. Initially, most of the scientific quest was focused on metallic systems [4]. However, in recent years,
EB phenomenon is also being explored and has rendered special importance to magnetic perovskite oxides [5-7]. The EB effect is commonly understood as the displacement of magnetic hysteresis loop along the field axis along with the increase in loop's width, which originates from the exchange interaction at the ferromagnetic (FM)/ antiferromagnetic (AFM) heterostructures during cooling in an external magnetic field [8]. It is then quite natural to expect an intrinsic EB effect in a single material responsible for the EB part of spintronic devices in a straightforward step [9].

More recently, an unexpected EB effect was observed in ferromagnetic ( $\mathrm{La}, \mathrm{Sr}) \mathrm{MnO}_{3}$ single, thin films on compressive strain substrates (such as $\mathrm{LaSrAlO}_{4}$ and $\mathrm{LaAlO}_{3}$ ), due to the emergence of a self-assembled spin glass-like phase of $\mathrm{LaSrMnO}_{4}$ at the film/substrate interface $[10,11]$. However, the EB was absent in this single manganite film with tensile stress. The $\mathrm{LaMnO}_{3}$ (LMO) bulk material exhibits AFM characteristic of the ground state, where Mn magnetic moments are arranged in an A-type AFM ordering [12]. However, the ferromagnetic insulating behavior is exhibited in LMO thin films, while the EB effect in single LMO films has been investigated under different oxygen pressures [13-16]. Regardless of extrinsic strain, the intrinsic EB effect in single LMO material is still a question of interest, which lacks experimental insight and in-depth investigation.

In this paper, the intrinsic EB effect in LMO single films can be observed no matter whether tensile or compressive strain is supplied by substrates. The X-ray absorption spectra (XAS) of Mn $L$-edge and $\mathrm{O} K$-edge, show that $\mathrm{Mn}^{2+}$ ions are exposed in the surface and could not be held responsible for canted ferromagnetic behavior for

\footnotetext{
${ }^{1}$ School of Chemistry and Materials Science, Key Laboratory of Magnetic Molecules and Magnetic Information Materials, Ministry of Education, Shanxi Normal University, Linfen 041004, China

${ }^{2}$ Research Institute of Materials Science of Shanxi Normal University \& Collaborative Innovation Center for Shanxi Advanced Permanent Magnetic Materials and Techonology, Linfen 041004, China

${ }^{3}$ School of Physics and Electronic Information, Shanxi Normal University, Linfen 041004, China

* Corresponding author (email: xuxh@sxnu.edu.cn)
} 
LMO thin film. The canted ferromagnetic properties are tunable through the rotation of $\mathrm{MnO}_{6}$ octahedron in the neighboring substrate. Furthermore, the antiferromagnetic order is mainly exhibited in the upper part of LMO films due to strain relaxation. As a result, the competition between the upper antiferromagnetic part and the bottom magnetic region can produce the EB effect in single LMO films.

\section{EXPERIMENTAL SECTION}

Epitaxial $\mathrm{LaMnO}_{3}$ films were prepared on different substrates, including $\mathrm{MgO}, \mathrm{Pb}\left(\mathrm{Mg}_{1 / 3} \mathrm{Nb}_{2 / 3}\right)_{0.72} \mathrm{Ti}_{0.28} \mathrm{O}_{3}$ (PMN$\mathrm{PT}), \mathrm{SrTiO}_{3}(\mathrm{STO}), \mathrm{LaAlO}_{3}(\mathrm{LAO})$, and $\mathrm{LaSrAlO}_{4}$ (LSAO), using pulsed laser deposition technique. Before the deposition, the substrates were heated to $725^{\circ} \mathrm{C}$ and held at that temperature for half an hour under the deposition pressure. This was done to ensure the release of unexpected contamination and reduce the additional oxygen vacancies on substrates. Deposition was carried out using a $248 \mathrm{~nm} \mathrm{KrF}$ excimer laser at $2 \mathrm{~Hz}$ under oxygen pressure of $100 \mathrm{mTorr}$. The number of pulses was adjusted to obtain the film thickness of 100 unit cells (u.c.). After growth, the samples were annealed in situ in 300 Torr oxygen atmosphere for one hour to improve the quality and reduce the inherent oxygen deficiency. Then, the samples were cooled to room temperature. Magnetic properties were measured in a vibrating sample magnetometer using PPMS (PPMS-VSM) magnetometry with the magnetic field applied in-plane along the (100) direction of the substrate within the temperature range of 5-300 K. The magnetization versus temperature $(M-T)$ measurements were performed after cooling from 300 to $5 \mathrm{~K}$ in zero-field-cooled (ZFC) or field-cooled (FC) processes. Conductivity was determined in a van der Pauw four-probe configuration using the physical property measurement system. The crystal structure and epitaxial orientation were characterized using conventional X-ray diffraction (XRD). The XAS measurements of $\mathrm{Mn} L$-edge and $\mathrm{O} K$-edge were performed at Beamline BL08U1A of the Shanghai Synchrotron Radiation Facility (SSRF) in total electron yield (TEY) mode. In order to avoid any contribution from the magnetic configuration of the sample, the temperature of the sample during XAS measurements was held at $300 \mathrm{~K}$.

\section{RESULTS AND DISCUSSION}

As shown in Fig. 1a, the clear (002) peaks of 100 u.c. LMO films are indicative of the high crystallinity of films and the absence of secondary phase on different substrates. Remarkably, the (002) peaks of LMO exist at the
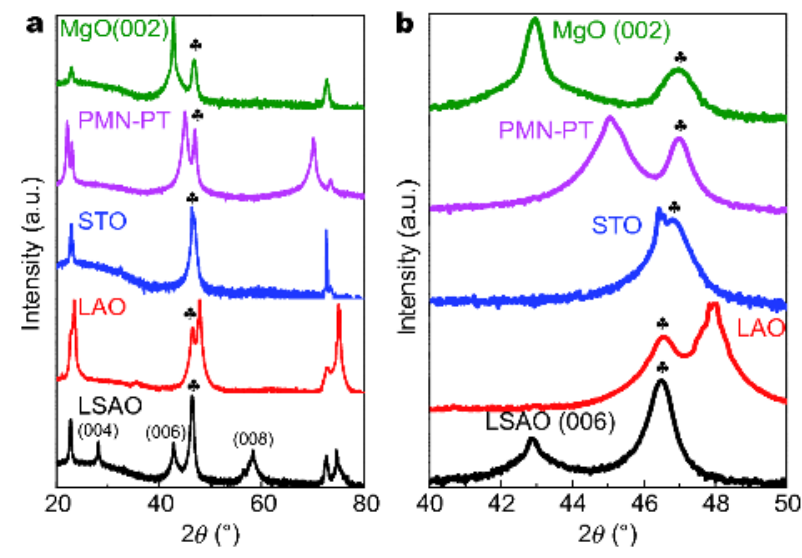

Figure 1 (a) XRD patterns for 100 u.c. LMO grown on MgO, PMN-PT, STO, LAO and LSAO substrates. (b) An expanded view around (002) reflections for clarity. The (002) peaks of LMO films are around the (002) substrate peak for MgO, PMN-PT, STO and LAO, except for the LSAO (006) substrate peak. The symbol * represents the (002) peak of LMO film.

right hand side of MgO, PMN-PT and STO substrates and at the left hand side of LAO and LSAO substrates. Therefore, MgO, PMN-PT, and STO substrates were used for tensile strain, while LAO and LSAO substrates were used for compressive strain. With respect to the Bragg reflections, the (002) peaks, shown in Fig. 1b, gradually shift towards lower growth angles from MgO to LSAO substrates, whereas the $c$-axis lattice constant in LMO films increases from 3.869 to $3.901 \AA$ [17]. The film/ substrate mismatch values for $\mathrm{MgO}, \mathrm{PMN}-\mathrm{PT}, \mathrm{STO}, \mathrm{LAO}$ and LSAO substrates are $\varepsilon_{0}=7.9 \%, 3.0 \%, 0.1 \%,-3.0 \%$ and $-3.9 \%$, respectively. In manganite materials, a few reports have focused on the half-filled $e_{\mathrm{g}}$ orbits of $\mathrm{Mn}^{3+}$, which guarantee the sensitivity of its magnetic and electric properties with respect to lattice distortion and orbital ordering through strain engineering [18].

Magnetic properties of the LMO thin films grown on different substrates under tensile to compressive strain are shown in Fig. 2. All the magnetic hysteresis loops of 100 u.c. LMO films were measured after field-cooling from room temperature to $5 \mathrm{~K}$ in a $\pm 5 \mathrm{kOe}$ field. For better comparison, the value of magnetization is represented in a unit of magnetization, called as Bohr magneton per Mn site. It is obvious that all the LMO films on different substrates show a ferromagnetic behavior and exhibit a shift of loop along the magnetic field axis. In general, the exchange field $\left(H_{\mathrm{EB}}\right)$ and coercivity $\left(H_{\mathrm{C}}\right)$ are quantified using the equations: $H_{\mathrm{EB}}=\left|H_{+}+H_{-}\right| / 2$ and $H_{\mathrm{C}}=\left|H_{+}-H_{-}\right| / 2$. The highlighted negative coercive $\left(H_{-}\right)$and positive coercive fields $\left(H_{+}\right)$of the film grown on different substrates indicate an asymmetry relative to 

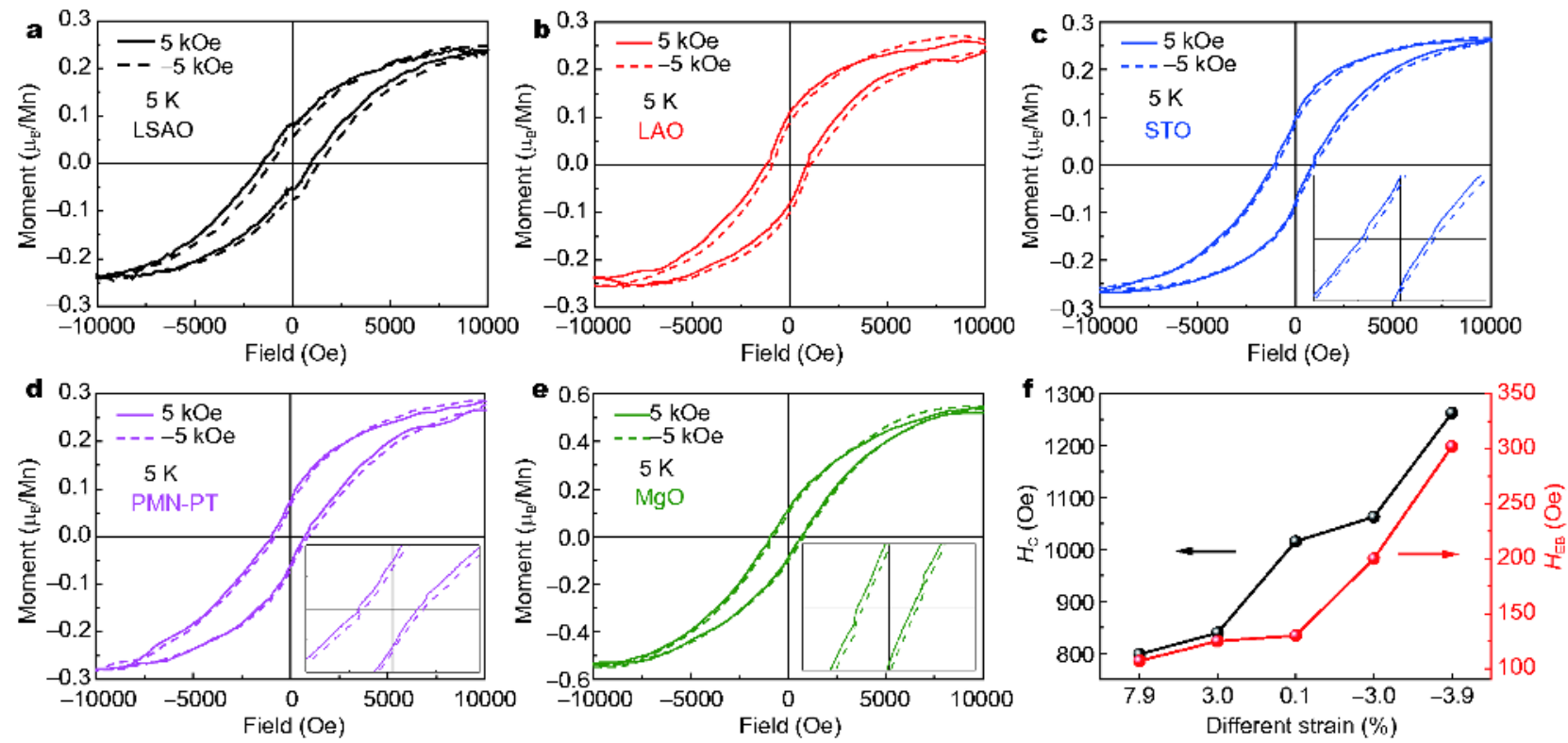

Figure 2 Hysteresis loops after \pm 5 kOe field-cooling from room temperature to $5 \mathrm{~K}$ for 100 u.c. LMO films on (a) LSAO, (b) LAO, (c) STO, (d) PMN$\mathrm{PT}$, and (e) $\mathrm{MgO}$ substrates. For clarity, only the data between $-10 \mathrm{kOe}$ and $10 \mathrm{kOe}$ are shown, whereas the actual measurements were conducted between -15 and $15 \mathrm{kOe}$. The region near the origin of loop is magnified to clearly show the extent of EB for STO, PMN-PT and MgO substrates. (f) The generalizations of different strain-dependent $H_{\mathrm{C}}$ (left axis) and $H_{\mathrm{EB}}$ (right axis) of LMO are also shown.

the zero-field [19]. After field-cooling at $+5 \mathrm{kOe}$, the shift in loop along the magnetic field axis towards the negative fields becomes the most prominent feature. In contrast, after cooling in the presence of a $-5 \mathrm{kOe}$ field, the loop is biased in the positive direction. This phenomenon shows that an A-type antiferromagnetic film has become ferromagnetic, and therefore, presents an obvious EB in itself [20].

The bias field $H_{\mathrm{EB}}$, which is defined as the absolute offset of loops along the field axis, is $302 \mathrm{Oe}$, whereas the coercivity $\left(H_{\mathrm{C}}\right)$ of LMO film on LSAO substrate is 1,263 Oe, as shown in Fig. 2a. For the LMO film on LAO substrate, as shown in Fig. $2 \mathrm{~b}$, the $H_{+}$and $H_{-}$are 863 and $-1,263$ Oe after $+5 \mathrm{kOe}$ field cooling process, indicating the EB field and the coercivity values of 200 and 1,063 Oe, respectively. For the LMO films on STO and PMN-PT substrates, the EB feature can be distinguished, as shown in the insets of Fig. $2 \mathrm{c}$ and $\mathrm{d}$. It can be observed that the shifts are 130 and $125 \mathrm{Oe}$, which are obtained from the magnified view of the zone around zero-field. Fig. 2e shows that, for the LMO film on $\mathrm{MgO}$ substrate, the $\mathrm{EB}$ is still around $107 \mathrm{Oe}$ and the saturation magnetization (Ms) is $5.4 \mu_{\mathrm{B}} / \mathrm{Mn}$, which has obviously increased compared with the values for other films. This apparent increase in Ms of LMO film on $\mathrm{MgO}$ substrate may be attributed to larger mismatch between the LMO film and the $\mathrm{MgO}$ substrate. As shown in Fig. $2 \mathrm{f}$, the value of $H_{\mathrm{C}}$ increases from 799 to $1,263 \mathrm{Oe}$, while that of $H_{\mathrm{EB}}$ increases from 107 to 302 Oe with regards to the change in strain values from $\mathrm{MgO}$ to LSAO. As a result, the ferromagnetic state and the unexpected EB effect are always exhibited in LMO thin films grown on different substrates. Here, the most eminent feature is that the EB in single LMO layer can be observed regardless of the contribution of tensile strain or compressive stress. This is very different from the results published previously, which show that the EB in single manganite is only observed under compressive stress [10]. Therefore, a deeper investigation of EB in single LMO films on different substrates is needed to achieve a better understanding of this system.

Herein, the origin of the EB exhibited in LMO films on different substrates and the LMO film on an LSAO substrate are considered. The corresponding temperature dependence of EB behaviors is shown in Fig. 3a, which are recorded after $+5 \mathrm{kOe}$ field cooling from room temperature to final measured temperature. It is obvious that, with the increase in temperature, the values of $H_{+}$and $H_{-}$ become closer to each other. As shown in the inset of Fig. 3a, the values of $H_{\mathrm{EB}}$ and $H_{\mathrm{C}}$ abruptly decay with the increase in temperature. However, $H_{\mathrm{EB}}$ almost vanishes just above $25 \mathrm{~K}$, which is referred to as the Blocking temperature. Blocking temperature is the highest temperature allowing the existence of EB [21]. In a previous 

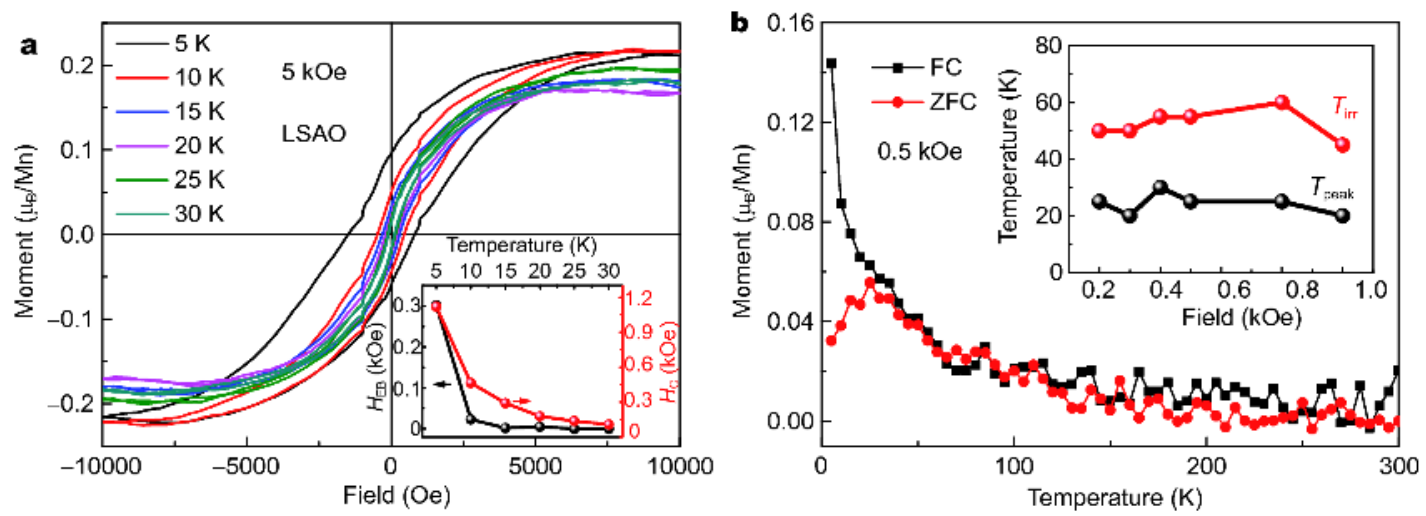

Figure 3 (a) Hysteresis loops of the 100 u.c. LMO films measured at different temperatures $(5,10,15,20,25$, and $30 \mathrm{~K})$ after $+5 \mathrm{kOe}$ field cooling. The summarized temperature dependences of $H_{\mathrm{EB}}$ (left axis) and $H_{\mathrm{C}}$ (right axis) after the same cooling processes are shown in the inset. (b) Temperature dependence of the magnetization curves measured at $0.5 \mathrm{kOe}$ after field cooling and zero-field cooling. The $T_{\mathrm{p}}$ and $T_{\text {irr }}$ are shown as a function of various fields $(H=0.2,0.3,0.4,0.5,0.8,1 \mathrm{kOe})$ in the inset.

study of single manganite under compressive stress, the spin glass behavior is reported to be the key factor responsible for EB effect. The magnetization versus temperature $(M-T)$ curves after FC and ZFC processes, measured in a 500 Oe field, are shown in Fig. 3b. Strikingly, the peak $\left(T_{\mathrm{P}}\right)$ in the $\mathrm{ZFC}$ curve and the bifurcation between the ZFC and FC curves below the temperature of irreversibility $T_{\text {irr }}$ are observed. As stated in a previous report, these two temperatures are observed in a spin glass based EB system, and decrease with the increase in measurement field, suggesting that the frozen state is suppressed by a strong field [19]. The $T_{\mathrm{P}}$ and $T_{\text {irr }}$ dependences of the measurement field are summarized in the inset of Fig. 3b. Notably, the two temperatures remain nearly constant with the increase in measurement field, thereby excluding the existence of spin glass feature. It should be pointed out that the $T_{\mathrm{P}}$ is close to the Blocking temperature, indicating that there is a collective freezing process of the moments of ferromagnetic clusters in LMO film, whereas the peak temperature can be regarded as the freezing temperature for FM clusters [22].

As reported previously, in manganite compounds, the ferromagnetic signals are generally induced by doubleexchange (DE) mechanism between $\mathrm{Mn}^{3+}$ and $\mathrm{Mn}^{4+}$ through oxygen ions [23]. In particular, the DE mechanism has successfully explained a ferromagnetic metallic ground state in doped manganite compounds. However, the LMO film is an insulating state and the ferromagnetic behavior has been due to another $\mathrm{DE}$ mechanism related to $\mathrm{Mn}^{2+}-\mathrm{O}-\mathrm{Mn}^{3+}$, where electrons are transferred from $\mathrm{Mn}^{2+}$ through hybridized oxygen $2 \mathrm{p}$ orbital to electronic configuration holes in the $e_{\mathrm{g}}$ band of $\mathrm{Mn}^{3+}$ [24]. Stoichiometrically, there are +3 ions each for
La and Mn in LMO. In the current experiments, the results show that this DE mechanism cannot be responsible for the canted ferromagnetic. The variations in Mn oxidation state between different samples could be evaluated using XAS measurements. It has been reported that, for lower $\mathrm{Mn}$ valences, the $\mathrm{Mn} L_{3}$ edge peak shifts towards lower energies, while the decrease in ratio of $L_{3}$ to $L_{2}$ intensities [25]. In the current measurements, Fig. 4a displays the XAS spectra about the $M n L_{3,2}$ edge for samples on different substrates. Neither an energy shift in the peak position nor an overall change in the spectral shape can be detected, indicating that the valence of $\mathrm{Mn}$ ions remains unchanged across the whole series of samples. However, it is obvious that all the spectra exhibit a small shoulder on the lower energy part of $L_{3}$ edge (as indicated by the arrow at around $640 \mathrm{eV}$ ). Valencia et al. $[26,27]$ reported that the evidence of the presence of $\mathrm{Mn}^{2+}$ ions as the peak of $640 \mathrm{eV}$ is attributed to the atmospheric $\mathrm{CO}$, which removes oxygen from the surface of oxide structure.

In addition, the $\mathrm{O} 2 \mathrm{p}$ states can provide information on Mn 3d occupancy due to hybridization between the $\mathrm{O} 2 \mathrm{p}$ and $\mathrm{Mn} \mathrm{3d}$ orbits, which is an indirect measure of the existence of $\mathrm{Mn}^{2+}-\mathrm{O}-\mathrm{Mn}^{3+}[28,29]$. The XAS spectra at the $\mathrm{O} K$-edge also appear in Fig. $4 \mathrm{~b}$. The region between 529 and $534 \mathrm{eV}$ (pre-edge) is attributed to the hybridization, which is caused by the $\mathrm{O} 2 \mathrm{p}$ and $\mathrm{Mn} 3 \mathrm{~d}$ orbitals. In particular, the peak marked by the thick arrow at $533 \mathrm{eV}$ is primarily caused by the $\mathrm{O} 2 \mathrm{p}$ hybridized orbital, providing circumstantial evidence of the occupancy of Mn 3d level [30]. The peak is absent in current measurements, suggesting that the magnetic properties in LMO thin films are not due to the $\mathrm{Mn}^{2+}-\mathrm{O}-\mathrm{Mn}^{3+} \mathrm{DE}$ 

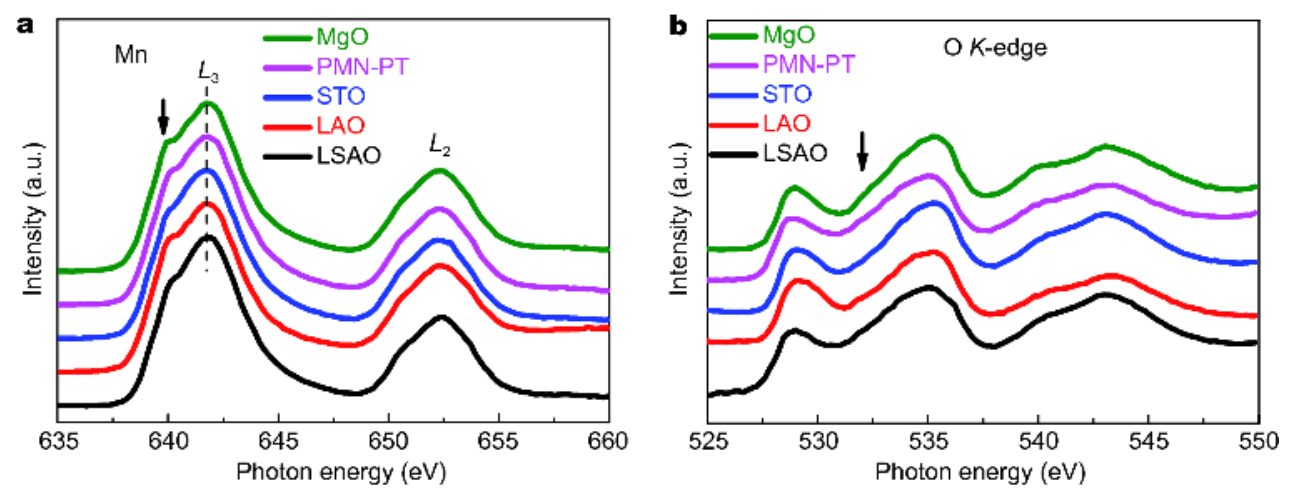

Figure 4 (a) Mn $L$-edge and (b) O K-edge XAS spectra measured using TEY mode for 100 u.c. LMO film on different substrates at room temperature. The two broad multiplets of $\mathrm{Mn} L$-edge are denoted as $L_{3}$ and $L_{2}$.

mechanism. Peng et al. $[16,26]$ has studied the O K-edge and pointed out that the intensity of $533 \mathrm{eV}$ peak increases with decrease in Mn valence, which is in contradiction to Valencia et al.'s results. In the current experiments, the $\mathrm{Mn}^{2+}$ is caused by the LMO film exposure to air. Therefore, the local ferromagnetic is not produced by $\mathrm{Mn}^{2+}-\mathrm{O}-\mathrm{Mn}^{3+} \mathrm{DE}$ mechanism, indicating that the origin of ferromagnetic state in LMO films should be further investigated for a better understanding.

As suggested by the theoretical studies, the different patterns of octahedral distortions that promote selective magnetic arrangements are due to strain-engineering caused by Jahn-Teller distortions or oxygen octahedral rotations [31]. This possibility has raised new research interest because strain induced FM has already been reported in other manganese thin films [32]. In this regard, it is worth stating that the epitaxial accommodation of highly distorted LMO structure on different substrates is not trivial. The LMO material is a Mott insulator with Atype antiferromagnetism, which results from cooperative Jahn-Teller distortions and leads to an anisotropic Mn-O$\mathrm{Mn}$ superexchange (ferromagnetic in-plane and antiferromagnetic out-of-plane as shown in Fig. 5a) [33]. The antiferromagnetism is slightly canted in the bulk, producing a weak magnetic moment of around $0.18 \mu_{\mathrm{B}}$ (where $\mu_{\mathrm{B}}$ is the Bohr magneton per u.c.), indicating that this is attributed to the antisymmetric DzyaloshinskiiMoriya (DM) interaction associated with the rotation of $\mathrm{MnO}_{6}$ octahedron [34]. The LMO films in the current experiments are grown on different substrates, providing additional stress in order to promote the canted magnetic ordering arrangement, as shown in Fig. 5b. Therefore, it is believed that the canted magnetic properties in LMO films can be attributed to $\mathrm{MnO}_{6}$ octahedral tilt and rotation, which are influenced by the strain-engineered
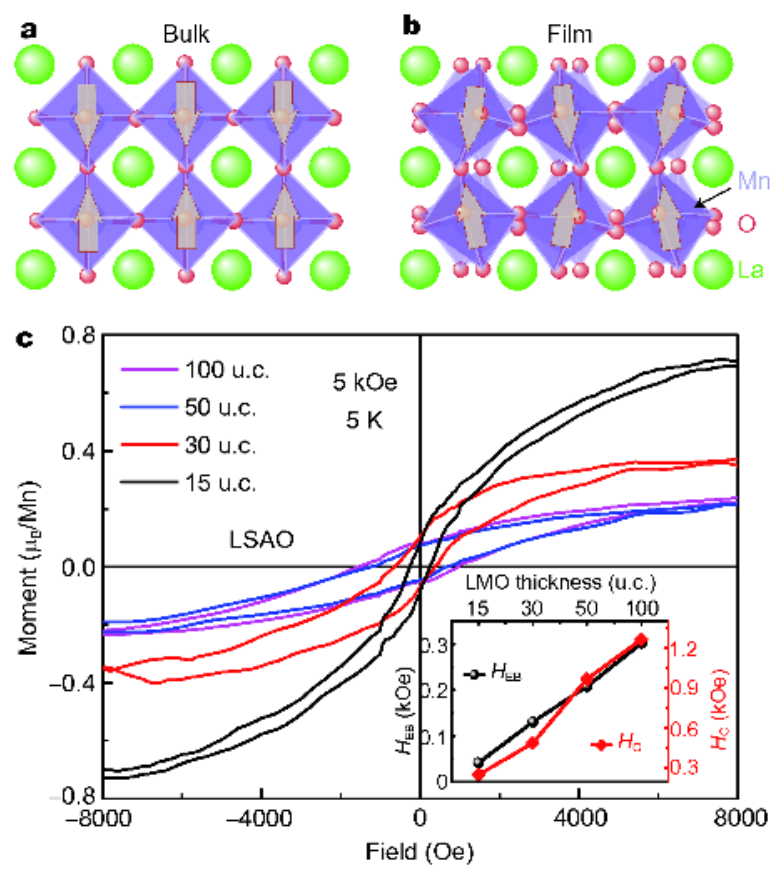

Figure 5 (a) The atomic structures of LMO bulk with no $\mathrm{MnO}_{6}$ octahedral rotations as A-AFM ordering. (b) The possible atomic structures of LMO film subject to constrained lattice mismatch, shown as canted FM ordering. (c) $M-H$ loops of LMO films deposited on LSAO substrate with various thicknesses $(100,50,30$, and 15 u.c.) measured at $5 \mathrm{~K}$ after $+5 \mathrm{kOe}$ field cooling from room temperature. The generalization of $H_{\mathrm{EB}}$ (left axis) and $H_{\mathrm{C}}$ (right axis) of LMO films are shown in the inset.

ferromagnetism, although accurate determination of its contribution is quite difficult. The LMO films on $\mathrm{MgO}$ substrates exhibit larger saturation magnetization, which is due to larger mismatch resulting in stronger oxygen octahedral rotation.

In an effort to further support the magnetic properties responsible for oxygen octahedral rotation, LMO films 
with different thicknesses (100, 50, 30, and 15 u.c.) were grown on LSAO substrate to investigate the dependence of thickness on magnetization. After cooling the LMO films to $5 \mathrm{~K}$ in a field of $+5 \mathrm{kOe}$, their hysteresis loops are recorded. The $H_{\mathrm{EB}}$ and $H_{\mathrm{C}}$ behaviors are plotted as a function of LMO thickness, and the results are shown in Fig. $5 \mathrm{c}$ and its inset. The saturation magnetization $\left(M_{\mathrm{S}}\right)$ increases from 0.21 to $0.72 \mu_{\mathrm{B}} / \mathrm{Mn}$ when the thickness reduces from 100 to 15 u.c., indicating the magnetic order plays a major role in thinner films. Therefore, it is inferred that the magnetic region favors the film/substrate interface, which contributes to strain $\mathrm{MnO}_{6}$ octahedral distortion. Due to the strain relaxation as a function of film thickness, the upper region mainly exhibits AFM order. The $H_{\mathrm{EB}}$ decreases monotonously from 302 to $41 \mathrm{Oe}$ as the film thickness decreases from 100 to 15 u.c., which is due to the decrease in AFM part in thinner films. Therefore, these two sub-layers affect each other, leading to the EB behavior observed in LMO films on different substrates.

\section{CONCLUSIONS}

In this work, the magnetic and structural properties of LMO thin films grown on different substrates are reported. The ferromagnetic state is attributed to the strain induced oxygen octahedral rotation rather than the presence of $\mathrm{Mn}^{2+}$ ions at the surface. The $\mathrm{EB}$ is caused by the pinning of ferromagnetic part in the bottom by the upper AFM region. It should be noted that the improvements introduced in this work can be useful in readily fabricating the strain induced spintronic devices.

Received 12 October 2018; accepted 20 December 2018; published online 11 January 2019

1 Meiklejohn WH, Bean CP. New magnetic anisotropy. Phys Rev, 1957, 105: 904-913

2 Tulapurkar AA, Suzuki Y, Fukushima A, et al. Spin-torque diode effect in magnetic tunnel junctions. Nature, 2005, 438: 339-342

3 Kools JCS. Exchange-biased spin-valves for magnetic storage. IEEE Trans Magn, 1996, 32: 3165-3184

4 Jiang Y, Nozaki T, Abe S, et al. Substantial reduction of critical current for magnetization switching in an exchange-biased spin valve. Nat Mater, 2004, 3: 361-364

$5 \mathrm{Yu}$ P, Lee JS, Okamoto $\mathrm{S}$, et al. Interface ferromagnetism and orbital reconstruction in $\mathrm{BiFeO}_{3}-\mathrm{La}_{0.7} \mathrm{Sr}_{0.3} \mathrm{MnO}_{3}$ heterostructures. Phys Rev Lett, 2010, 105: 027201

$6 \mathrm{He} \mathrm{C}$, Grutter $\mathrm{AJ}, \mathrm{Gu} \mathrm{M}$, et al. Interfacial ferromagnetism and exchange bias in $\mathrm{CaRuO}_{3} / \mathrm{CaMnO}_{3}$ superlattices. Phys Rev Lett, 2012, 109: 197202

7 Ding JF, Lebedev OI, Turner S, et al. Interfacial spin glass state and exchange bias in manganite bilayers with competing magnetic orders. Phys Rev B, 2013, 87: 054428

8 Yu T, Ning XK, Liu W, et al. Exchange bias effect in epitaxial
$\mathrm{La}_{0.67} \mathrm{Ca}_{0.33} \mathrm{MnO}_{3} / \mathrm{SrMnO}_{3}$ thin film structure. J Appl Phys, 2014, 116: 083908

9 Kiwi M. Exchange bias theory. J Magn Magn Mater, 2001, 234: 584-595

10 Cui B, Song C, Wang GY, et al. Strain engineering induced interfacial self-assembly and intrinsic exchange bias in a manganite perovskite film. Sci Rep, 2013, 3: 2542

11 Cui B, Song C, Li F, et al. Tuning the entanglement between orbital reconstruction and charge transfer at a film surface. Sci Rep, 2014, 4: 4206

12 Choi WS, Jeong DW, Jang SY, et al. $\mathrm{LaMnO}_{3}$ thin films grown by using pulsed laser deposition and their simple recovery to a stoichiometric phase by annealing. J Korean Phy Soc, 2011, 58: 569574

13 Ritter C, Ibarra MR, De Teresa JM, et al. Influence of oxygen content on the structural, magnetotransport, and magnetic properties of $\mathrm{LaMnO}_{3+\delta}$. Phys Rev B, 1997, 56: 8902-8911

14 Hou YS, Xiang HJ, Gong XG. Intrinsic insulating ferromagnetism in manganese oxide thin films. Phys Rev B, 2014, 89: 064415

15 Zhou G, Yan Z, Bai Y, et al. Exchange bias effect and orbital reconstruction in (001)-oriented $\mathrm{LaMnO}_{3} / \mathrm{LaNiO}_{3}$ superlattices. ACS Appl Mater Interfaces, 2017, 9: 39855-39862

16 Peng JJ, Song C, Cui B, et al. Exchange bias in a single $\mathrm{LaMnO}_{3}$ film induced by vertical electronic phase separation. Phys Rev B, 2014, 89: 165129

17 Lee JS, Arena DA, Yu P, et al. Hidden magnetic configuration in epitaxial $\mathrm{La}_{1-x} \mathrm{Sr}_{x} \mathrm{MnO}_{3}$ films. Phys Rev Lett, 2010, 105: 257204

18 Grutter AJ, Vailionis A, Borchers JA, et al. Interfacial symmetry control of emergent ferromagnetism at the nanoscale. Nano Lett, 2016, 16: 5647-5651

19 Wu L, Ma J, Ma J, et al. Exchange coupling-induced uniaxial anisotropy in $\mathrm{La}_{0.7} \mathrm{Sr}_{0.3} \mathrm{MnO}_{3}$ thin films. Sci Bull, 2016, 61: 157-162

20 Gao Y, Wang J, Wu L, et al. Tunable magnetic and electrical behaviors in perovskite oxides by oxygen octahedral tilting. Sci China Mater, 2015, 58: 302-312

21 Gibert M, Zubko P, Scherwitzl R, et al. Exchange bias in $\mathrm{LaNiO}_{3}$ $\mathrm{LaMnO}_{3}$ superlattices. Nat Mater, 2012, 11: 195-198

22 Tian YF, Ding JF, Lin WN, et al. Anomalous exchange bias at collinear/noncollinear spin interface. Sci Rep, 2013, 3: 1094

23 Orgiani $\mathrm{P}$, Aruta $\mathrm{C}$, Ciancio R, et al. Enhanced transport properties in $\mathrm{LaxMnO}_{3-\delta}$ thin films epitaxially grown on $\mathrm{SrTiO}_{3}$ substrates: The profound impact of the oxygen content. Appl Phys Lett, 2009, 95: 013510

24 Galdi A, Aruta C, Orgiani P, et al. Magnetic properties and orbital anisotropy driven by $\mathrm{Mn}^{2+}$ in nonstoichiometric $\mathrm{La}_{x} \mathrm{MnO}_{3-\delta}$ thin films. Phys Rev B, 2011, 83: 064418

25 de Jong MP, Bergenti I, Dediu VA, et al. Evidence for $\mathrm{Mn}^{2+}$ ions at surfaces of $\mathrm{La}_{0.7} \mathrm{Sr}_{0.3} \mathrm{MnO}_{3}$ thin films. Phys Rev B, 2005, 71: 014434

26 Valencia S, Gaupp A, Gudat W, et al. Mn valence instability in $\mathrm{La}_{2 / 3} \mathrm{Ca}_{1 / 3} \mathrm{MnO}_{3}$ thin films. Phys Rev B, 2006, 73: 104402

27 Yi D, Liu J, Okamoto S, et al. Tuning the competition between ferromagnetism and antiferromagnetism in a half-doped manganite through magnetoelectric coupling. Phys Rev Lett, 2013, 111: 127601

28 Galdi A, Aruta C, Orgiani P, et al. Electronic band redistribution probed by oxygen absorption spectra of $\left(\mathrm{SrMnO}_{3}\right)_{n}\left(\mathrm{LaMnO}_{3}\right)_{2 n}$ superlattices. Phys Rev B, 2012, 85: 125129

29 Valencia S, Gaupp A, Gudat W, et al. Impact of microstructure on the Mn valence of $\mathrm{La}_{2 / 3} \mathrm{Ca}_{1 / 3} \mathrm{MnO}_{3}$ thin films. Phys Rev B, 2007, 75: 184431 
Zhou G, Song C, Bai Y, et al. Robust interfacial exchange bias and metal-insulator transition influenced by the $\mathrm{LaNiO}_{3}$ layer thickness in $\mathrm{La}_{0.7} \mathrm{Sr}_{0.3} \mathrm{MnO}_{3} / \mathrm{LaNiO}_{3}$ superlattices. ACS Appl Mater Interfaces, 2017, 9: 3156-3160

31 Lee JH, Delaney KT, Bousquet E, et al. Strong coupling of JahnTeller distortion to oxygen-octahedron rotation and functional properties in epitaxially strained orthorhombic $\mathrm{LaMnO}_{3}$. Phys Rev B, 2013, 88: 174426

32 Chou H, Tsai MH, Yuan FP, et al. Effects of strain on the electronic structures and TC's of the $\mathrm{La}_{0.67} \mathrm{Ca}_{0.33} \mathrm{MnO}_{3}$ and $\mathrm{La}_{0.8} \mathrm{Ba}_{0.2} \mathrm{MnO}_{3}$ thin films deposited on $\mathrm{SrTiO}_{3}$. Appl Phys Lett, 2006, 89: 082511

33 Wang XR, Li CJ, Lü WM, et al. Imaging and control of ferromagnetism in $\mathrm{LaMnO}_{3} / \mathrm{SrTiO}_{3}$ heterostructures. Science, 2015, 349: 716-719

34 Coey JMD, Viret M, von Molnár S. Mixed-valence manganites. Adv Phys, 1999, 48: 167-293
Acknowledgements The authors acknowledge Beamline BL08U1A in Shanghai Synchrotron Radiation Facility (SSRF) and Beamline BL12-a in National Synchrotron Radiation Laboratory (NSRL) for X-ray absorption measurement at room temperature. This work was financially supported by the National Key R\&D Program of China (2017YFB0405703), the National Natural Science Foundation of China $(51871137,61434002$ and 51571136), and the Special Funds of Sanjin Scholars Program.

Author contributions Zhou G designed and performed the experiment, analyzed the results, and wrote the manuscript. Ji H performed the experiment. Bai Y and Quan Z helped analyze the results and modify the manuscript. $\mathrm{Xu} X$ supervised the work and revised the manuscript. All authors read and approved the final manuscript.

Conflict of interest The authors declare that they have no conflict of interest.

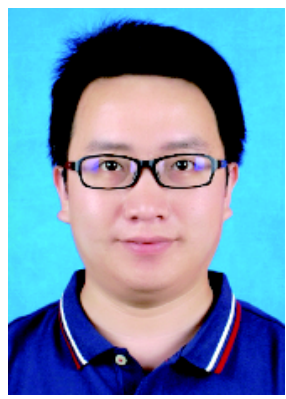

Guowei Zhou obtained his PhD degree from Shanxi Normal University in 2018. He is currently a lecturer in Prof. Xu's group at Shanxi Normal University. His current research interest focuses on emerging phenomena and exotic physical behaviors in perovskite complex oxides films and heterostructures.

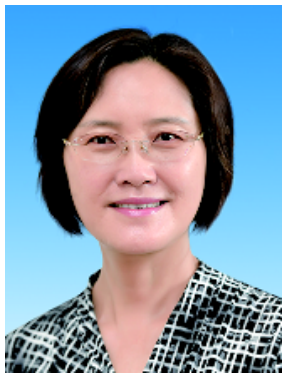

Xiaohong Xu received her PhD in material science and engineering from Xi'an Jiaotong University, China in 2001. From 2001-2006, she was in Huazhong University of Science and Technology, China, the University of Sheffield, UK, and Tohoku University, Japan as a postdoc or research fellow. Her research interest includes oxide semiconductor spintronics, magnetic recording media and interface physics of heterostructures. She is a Distinguished Young Scholar awarded by the National Natural Science Foundation of China.

\section{反铁磁 $\mathrm{LaMnO}_{3}$ 薄膜中应力调控的交换偏置现象}

周国伟 ${ }^{1,2}$, 姬慧慧 ${ }^{2}$, 白宇浩 ${ }^{1,3}$, 全志勇 ${ }^{1,2}$, 许小红 ${ }^{1,2 *}$

摘要 本文用脉冲激光沉积系统外延生长了 $\mathrm{LaMnO}_{3}(\mathrm{LMO})$ 薄膜, 研究了拉应力和压应力对薄膜磁学性质的影响, 发现在拉应力和压应力 的衬底上, 外延生长这种 $\mathrm{A}$ 型反铁磁 $\mathrm{LMO}$ 薄膜, 均可出现交换偏置现象. 这是因为外应力导致衬底与薄膜界面处的 $\mathrm{MnO}_{6}$ 氧八面体发生转 动, 从而使临近衬底的LMO薄膜下层出现了净磁矩表现铁磁性; 而那些远离衬底的LMO薄膜上层则由于外应力的释放, 仍保持原有的反 铁磁性. 因此, LMO薄膜中自发的交换偏置现象, 源于铁磁性与反铁磁性之间的交换耦合作用. 这种在单一LMO薄膜中实现交换偏置的现 象, 为自旋阀器件的优化设计提供了一种新方法. 